\title{
The informational physical model and fundamental problems in physics
}

\author{
Sergey V. Shevchenko ${ }^{1, *}$ and Vladimir V. Tokarevsky ${ }^{2}$ \\ ${ }^{1}$ Institute of Physics of NAS of Ukraine, Pr. Nauki, 46, Kiev-28, Ukraine, ret. \\ ${ }^{2}$ Professor ret., Pr. Nauki, 46, Kiev-28, Ukraine \\ *Corresponding: sshev1g@gmail.com
}

\begin{abstract}
This article is the review of results that were obtained at 2007-2021 years development of "The Information as Absolute" concept and the informational physical model, which is based on the concept; including a number of fundamental physical problems are briefly considered in framework of the conception and the model. Recently in physics there are several publications, that present lists of the problems. However, those lists are essentially incomplete, for at least two reasons. Firsts of all, a number of phenomena are studied traditionally by philosophy, and so corresponding problems are usually considered to be "metaphysical". However, they relate also to some concrete physical phenomena. For example, physics evidently studies Matter, and so the metaphysical problems "what is ontology of Matter", "what is "Space", "Time" and a few other physical phenomena and notions as well, are really a Meta-physical problem "what does physics study?" There are other fundamental physical problems, which are not considered as such in physics, and are absent in the "fundamental problems lists". Those include the problems, which really exist, yet are incorporated into standard physical theories, and so are fundamental "implicitly", which in physics are "solved by default". Note, though, that a number of "Metaphysical", and concrete fundamental, problems in detail are considered in the paper "The Informational Conception and Basic Physics", arXiv:0707.4657, v5 (2021), so in this paper some selected common physical problems are considered, and, besides, the problems "What is Life" and "Cosmology".
\end{abstract}

Key words: informational physics, Matter, Consciousness, life, fundamental physical problems, fundamental Nature forces, relativity, cosmology, spacetime, experimental testing, quantum mechanics, particles, antiparticles.

PACS numbers: 01.70.+w, 03.30.+p, 04.80.Cc 14.80.Hv, 12.20.-m, 11.15.Kc, 11.80.Fv 03.50; 12.60.-i; 12.90.+b; 14.60.Cd; 03.70.

\section{Introduction}

In this article a number of fundamental physical problems are briefly considered in framework of the "The Information as Absolute" concept and the informational physical model, which is based on the concept, that were developed in 2007-2020 [1]-[15] .

By now, there exist a number of publications, where the authors formulate some lists of fundamental physical problems, for example, [16], [17], the corresponding Wikipedia article, etc., analogously to Hilbert's presentation of twenty-three problems in mathematics at the International Congress of Mathematicians in Paris in 1900 [16]. The 
lists of problems are quite similar. Correspondingly in this paper we consider some problems in one of rather complete list in [17], which are considered also in the main text, and briefly commented upon in the Appendix.

Notably, the problem lists in such publications essentially are incomplete, by two reasons. Firsts of all there are a number of physical phenomena that are studied traditionally by philosophy, and so usually are considered "metaphysical". They relate, nonetheless, to physical phenomena - for example physics evidently studies Matter, and so the metaphysical problem "what is ontology of Matter" is really a Meta-physical problem "what does physics study" as well.

The metaphysical problem "what is ontology of Consciousness" also relates to physics directly, starting from the physical problem "why and how physical measurements and interpretations of the measurements are sometimes adequate to the objective reality"? Besides, this problem was actual on first stages of development of the quantum mechanics, and is rather actual now.

Such metaphysical problems as what are the fundamental phenomena/notions "Space" and "Time", and a number of others, are really the fundamental physical problems as well.

Other problems that really are fundamental physical problems, but are not considered as such in physics, and so in the "fundamental problems lists", are the problems incorporated into standard physical theories, and thus are "implicitly" fundamental. For example, in [17] the problem "Why are the particles of ordinary matter copied twice at higher energy" is pointed. However, the problem "what are particles" precedes that, and seems as evident that only after solving that last problem it would be possible to obtain the rational answer for the first problem, etc. However, the list in [17] does not contain the latter problem, which - and a number of others - in physics are "solved by default", despite that really, rather evidently, exist.

A number of "Meta-physical", and concrete fundamental, problems in detail are considered in the arXiv paper "The Informational Conception and Basic Physics" v5 2021 year version [5a], so in this paper some selected common physical problems are considered in detail, and, besides, the problems "What is Life" and "Cosmology".

Note, that the concept and the model are discussed and some papers are commented at ResearchGate Net, thus the corresponding ResearchGate URLs are given in the Sec. "References", besides the standard reference data.

Further, correspondingly, in this paper we present the basic assertions, postulates and assumptions, which are obtained at solving and clarifying of the utmost fundamental philosophical and physical "Meta-problems", which are the utmost fundamental base of the informational physical model, brief description of the fundamental problems that are in detail considered in [5a], and consideration of a few common fundamental physical problems. Besides in Appendix brief comments to the published fundamental physical problems list "Life, the universe, and everything - 42 fundamental Questions" [17] are given. 


\section{Meta-problems}

\section{1 "What is "Information"}

Really the phenomenon/notion "Information" in philosophy and sciences remains to be principally transcendent - neither philosophy nor any science define "Information" substantively enough, all what exists is/are definitions of only specific traits/properties of Information (more see [3]), starting from the evident from everyday practice traits that "information" is

"(Philosophical encyclopedia) "Information (lat. "informatio" - an examination, a notion, a concept): 1) a report, a notification about a state of affairs or about something else that is transmitted by a person; 2) decreased, removed uncertainty as a result of the communication obtained; 3) a notation inherently relating to a control; signals and their syntactic, semantic and pragmatic parameters; 4) transmission, reflection of the variety of any objects and processes (of alive and non-alive nature)";

- i.e. briefly "information is some data". That is evident tautology, however this tautology is inevitable in mainstream philosophy and science, since really the Information is absolutely fundamental and common phenomenon/notion, and so principally cannot be defined through some more common notions.

The correct scientific elaboration of the problem "what is "Information"" was made in the "The Information as Absolute" concept, [3], which was developed in 2007-2017,

- where it is rigorously proven that nothing else exists besides some informational patterns/systems of the patterns that are elements of the absolutely fundamental and absolutely infinite "Information" Set.

Which (the Set) exists absolutely objectively really, because it fundamentally, logically cannot be non-existent, and so is absolutely eternal, having no Beginning and no End.

The phenomenon "Information" and the Set have a number of fundamental properties, which are considered in [3], so more see the referenced paper, here note only that Information is extremely bifurcational and paradoxical phenomenon, which principally cannot be formalized in any theory, and so, for example, existent in sciences a few "theories of information", i.e. "Shannon informational theory", a few theories in cybernetics, describe only some essentially limited informational structures.

In the concept the utmost common definition of the absolutely fundamental ${ }^{1}$ phenomenon, "Information" is:

"Information is something that is constructed in accordance with the set/system of absolutely fundamental Rules, Possibilities, Quantities, etc. — the set/system "Logos" in the concept".

Or, by other words, the "Logos" set elements "make something to be information".

\footnotetext{
${ }^{1}$ Here and further "absolutely fundamental" relates to phenomena/notions that exist and are valid on whole "Information" Set, when ("simply") "fundamental" relates to phenomena/notions that are fundamental in Matter and "consciousness on Earth", including human consciousness, and in the natural and social sciences.
} 
A few examples of the "Logos" elements, which will be, since they do not have rational definitions in the mainstream science, and are scientifically defined in [3] and [5a], used further in this paper are, first of all, "Space", "Time", "Logical Rules", "Energy", "Change". Including that the Rules/Possibilities "Space" and "Time" actualize themselves as concrete spaces of concrete Set's elements,

- and mostly the concrete spaces have a number of the "space dimensions", every of which relates to concrete degree of freedom at the element's state changes, whereas at all changes of states of all dynamical elements in the Set, the changing states are placing in unique, fundamentally universal for all elements, time dimension.

\section{2. "What are "Matter" and "Consciousness"}

The utmost fundamental in the mainstream science "simply" Meta-phenomena/notions "Matter" and "Consciousness" are fundamentally transcendent in mainstream philosophy, and so the philosophy is composed by two fundamentally different doctrines, which have numerous sub-doctrines, schools, etc., "Materialism" and "Idealism", which really are nothing else than systems of transcendent beliefs:

- materialists truly believe in some transcendent "Matter", which for some transcendent reasons, and by some transcendent way, exists eternally; and is, as that follows from the observations, again for some for some transcendent reasons, and by some transcendent way, some evidently well logically organized system;

- idealists truly believe in some transcendent "Idea" ("Spirit", "Consciousness", etc.) which for some transcendent reasons, and by some transcendent way, exists eternally; though in this doctrine the fact that everything looks as evidently logically organized system follows from that is result of action of "conscious" "Idea", etc., - though it is evidently in this case to define - what is "conscious", what is again fundamentally impossible in the both doctrines; and so the transcendence of Idealism really isn't lesser than of Materialism

In the concept both, "Matter" and "Consciousness", are utmost commonly scientifically defined - "Matter" and "Consciousness" absolutely for sure are nothing else than some informational systems - the Set's elements, so are made from the same stuff "Information", and in accordance with the same "Logos" set's elements; whereas "Information" (and most of "Logos" elements as well aren't) isn't transcendent, and it, and so any informational structure as well, can be principally rationally cognizable.

Note here also, that from the above it follows that any informational system of elements is always something like "computer+program shell" system, where "hardware" is the elements, and "program shell" is the concrete the system's basic set of laws/links/constants, in accordance with the elements interact composing just this system, exchanging at that by some informational messages, which use concrete language in the system.

Including "Matter" and "Consciousness" are some systems, which, however, have fundamentally different basic sets of the laws/links/constants, and so are fundamentally different; the main difference is in that Matter is logically closed in the Set system, which so practically doesn't interact with other the Set's elements and thus is essentially stable system; 
- whereas Consciousness is fundamentally open in the Set system, and, as that is one of the utmost specific properties of just any consciousness in the Set, is that consciousness principally is able to obtain and analyze any information in the "Information" Set; at that, however, because of consciousness has fundamentally limited capabilities at obtaining and processing of the principally infinite in this case information, every result of the processing is always at least partially uncertain;

- and, at that, if a consciousness obtains some information, about which she hasn't some earlier information, the consciousness assigns to this information the label "this information is non-understandable", and further, if that is necessary, or that is interesting ["Curiosity" is another utmost just specific property/ resident utility in the "shell" of any consciousness], studies the Set's element, from which this information is obtained - again "no understanding" state is just fundamental specific state of any consciousness.

All that is in principal contrast to what happens in Matter, where every of Matter's elements, i.e. particles, bodies, fields, cosmological objects, always completely knows all Matter's laws/links/constants, so at interactions uses/exchanges by only true information, and behaves after obtaining some concrete message again in complete accordance with the basic Matter set above. Or, by another words, since the set "is written" in every Matter's element, Matter isn't some "whole" computer, it is an automaton,

- whereas any Consciousness version is "whole" computer, i.e. her hardware and program shell contain some "BIOS", "processor", "random access memory", and some specific utilities that organize work of the whole consciousness' s functional modules.

Finally, here note, that, though "Matter" and "Consciousness" are fundamentally different systems, whereas physics really studies only Matter, and so really the "consciousness problem" isn't a physical problem, nonetheless understanding of "what consciousness is" is necessary for physicists for a number of reasons. First of all, in this case we have answers on the really main epistemological - and practically so important in any science - question "so what studies of what?", and "why the first what sometimes adequately, and sometimes illusorily, to the objective reality studies the second what?"

Both these questions principally cannot be answered in framework of the mainstream, since in the mainstream both the whats are fundamentally transcendent, however in the concept the answers are natural: in spite of that consciousness, including the "homo sapiens sapiens" version, and Matter, are fundamentally different, however, since both are made from nothing besides "Information", and absolutely obligatorily in accordance with the same "Logos" set,

- there is nothing surprising in that one informational system, which is able to obtain from, and logically analyze information about, other informational system, makes that correctly, and sometimes incorrectly - that principally doesn't differs, say, from the case when a human decode information that was created by other human, say, when some linguists decode hieroglyphs that were written on some non-existent now languages. 
Besides historically the "consciousness problem turned out to be an - and rather popular - fundamental physical problem, and so it is pointed practically in all "lists of fundamental physical problems" above, being formulated usually, though, as "what is Life" problem; and, besides, historically the "consciousness problem" appeared in well known physical problem of the role of observer at quantum mechanics measurements; so "consciousness problem" it is considered also in this paper in corresponding "What is "Life" section below.

\subsection{What is "Life"}

As that was pointed above the two known now fundamental informational systems Matter" and "Consciousness" are fundamentally different. Currently humans know only one Consciousness' version, "the consciousness on Earth", diverse versions of which every living being on Earth, including humans, have. The main differences - and similarities - between a Matter and any Consciousness in the Set are pointed as well both systems are made in accordance with the same "Logos" elements, both are some "computer+program" systems, where similar exchange by concretely logically organized information between the systems' elements proceeds;

- however these systems are fundamentally different since are based on fundamentally different sets of the basic laws/links/constants; and so, though in Matter all/every elements, say, every electron, know physics absolutely completely, what any human never will do, however, at that, all/any material objects/structures fundamentally don't know - and fundamentally aren't able to know - anything else, thus everything in Matter fundamentally isn't, and never can be, “conscious".

Correspondingly every of both, Matter and Consciousness, exists and changes in essentially different spaces; say, if in Matter it is possible to establish some etalon for measurement of bodies' lengths and distances, say, "meter", but nobody now knows, say - how many meters long a human's thought is in a consciousness space dimension.

Though consciousness on Earth operates also in Matter's space, when she governs, using some unknown forces, practically material living beings organisms, including systems "body+brain". Both corresponding spacetimes share the one true time dimension which is fundamentally obligatorily common for all dynamical patterns/systems in whole Set (more about what are space/time/spacetime see [5a] and below).

Thus, there principally cannot be some "emergence" of any consciousness from any material structure, as that is, as a rule, assumed in many existent now "theories" "models", "solutions" of the "mind-body problem", etc., in neuroscience and physics. Really the informational system "the consciousness on Earth" could, in principle, exist in the Set in parallel with possible Matter's Creator even before Beginning of Matter.

However, because the consciousness is a principally open informational system, the "life" of such system is rather cumbersome in the unstable, and possibly destructive, environment of the Set. So seems a few billions of years ago this consciousness version has used an opportunity to make some material house from some stable Matter's atoms, first of all as a stable residence and source of energy at operating and development. Thus Life rather probably appeared on Earth (though we cannot exclude now that this 
consciousness version was developed and created by some other Consciousness in the Set, for example by with rather non-zero probability existent Creator od Matter).

After that, "the consciousness on Earth" developed the practically material residence in accordance with seems evidently observed trend "more and more outside Matter into other Set's regions", up to the "homo sapiens sapiens" version. That one has well developed ability to obtain and to process information in the highest, "mind mode", mode of operation, when information is processed abstractly i.e. in some cases without direct relation to what happens in Matter, or somewhere else in the Set.

However, the consciousnesses ability to affect material structures is extremely weak, at least for ordinary human consciousnesses, including most of physicists; and so really there is no some "observer problem" in physics:

- at any experiment a studied, including a quantum mechanical, material object/system/process interacts with human material instruments, mostly as observed " $\psi$-function collapses", only in rigorous consistence with the laws/links/constants that act only in Matter, when on the QM depth everything in Matter, independently on - in humans experiments or not, constantly happens as endless chains of the " $\psi$-function collapses", etc.,

- and all that, again, happens without any dependence on whether an "observer" exists or not at all; including the collapse of Schrödinger cat wave function would happen without any relation - some observer opens or not the box.

More about "the consciousness on Earth" see in the first approximation functional model of the consciousness [14], [15].

\section{General physics and quantum mechanics fundamental problems that are considered in [5a]}

\subsection{Common and general physics problems}

As that was pointed in Introduction above a number of essentially concrete fundamental problems are considered in detail in [5a], including the Meta-problems of what are the "Logos" set elements, i.e.

3.1.1- what are actualizations the Logos elements "Space", "Time", "Energy" in the concrete informational system "Matter" as the Matter's space/time/spacetime and energy; including it is shown that the existent in physics definitions of common physical variable/QM observable “time”, i.e. the Newton's [18]

"...Absolute, true and mathematical time, of itself, and from its own nature flows equably without regard to anything external, and by another name is called duration ....",

- and definitions in the relativity theories [29], are wrong, time absolutely fundamentally doesn't flow anywhere, and so, e.g., cannot be "dilated", including really there is no some "time arrow" [19], etc.;

3.1.2 - is Matter's spacetime absolute or not [24], [25] - it is rigorously proven that the spacetime is absolute; 
3.1.3 - what is "Inertia" and "inertial mass"; including how inertia/inertial mass and energy are fundamentally linked in Matter, despite that are fundamentally different phenomena;

3.1.4 - what is Matter's logical base; and how the logical base is actualized in Matter;

3.1.5 - what are particles;

3.1.6 - what are antiparticles;

3.1.7 - why neutrinos have rest masses;

3.1.8 - what is the physical sense of Lorentz transformations and special relativity theory, including what are Lorentz factor, "length contraction" , and "relativity of simultaneity" [24], [28], [29], [30], [31];

3.1.9 - what is the physical sense of the main equations of the fast bodies dynamics [29].

\subsection{Informational approach and $Q M$}

3.2.1 - why in Matter quantum effects exist at all;

3.2.2 - why does the QM postulate exist that all given type particles are identical, and why is it adequate to the reality;

3.2.3 - what is the wave-particle duality.

3.2.4 - what is particle's spin;

3.2.4 - what is the Planck constant, physical action, Uncertainty principle, least physical action principle.

\subsection{Possible rational clarifications of some general physics problems}

In the sections 3.1 and 5.2 above a number of practically for sure already solved problems are listed, however in framework of the informational model rational physical approaches at solutions of some other general physics fundamental problems, rather probably are at least essentially clarified, whereas in recent physics these problems really remain transcendent. First of all that relates to mediation of the fundamental Nature forces in complex systems - and, of course, to the problem "what is a fundamental Nature force?" at all; more concretely:

3.3.1 - what are fundamental Nature forces and charges;

3.3.2 - what is a Force charge of a particle;

3.3.3 [developed in framework of the whole model] Initial model of Gravity Force, including rational clarifying of the problems:

3.3.3.1 - why the fundamentally different inertial and gravitational masses are equivalent at least at statics; 


\subsubsection{2 - what is quantum Gravity;}

3.3.3.3 - how the quantum nature of Gravity can be experimentally observed;

3.3.4 [developed in framework of the whole model] Initial model of Electric Force, including rational clarifying of the problems:

3.3.4.1 - why the strengths of Gravity and Electric Forces are as they are;

3.3.4.2 - why magnetic monopole doesn't exist;

3.3.4.3 - what is the fine structure constant, and what is the physical sense of equation $\alpha \hbar c=e^{2} / 4 \pi \varepsilon_{0}$ in quantum mechanics;

3.3.4.4 - some notes about what are virtual particles in QED.

3.4 Planck mass particles and their role in Matter, though that more in detail is considered in this paper section 6 "Cosmology"

3.5. Experiments that are proposed for testing of the model

3.5.1 - observation of the quantum nature of Gravity [6];

3.5.2 - solution of the Poincaré problem [23] of experimental observation of the absolute motion of a body in the absolute Matter's space [9];

3.5.3 - observation of that photons at propagating in Gravity field between point with different potentials gain/lose energy, whereas that contradicts with the general relativity postulate, which states that photons propagate along geodesics with constant energy/frequency [10] (viXra file)

\section{Some additional remarks}

So more about the fundamental problems in the section 3 above see [5a], however for convenience at understanding of what is below in this paper it looks as is necessary to clarify briefly some basic points that are considered in this paper.

\section{1 "What is Matter's logical base"}

In the model the Matter's logical base follows basing on a few basic points:

- first of all on that Matter is for sure some informational system/an element of the Set and so for sure has some logical structure, whereas from existent experimental data follows that Matter indeed is such - and well logically organized - system, including so mathematics turns out to be extremely effective tool at analysis of experimental data,

- on the scientific definitions of the fundamental phenomena/notions, first of all, "Information", "Matter", "Space", "Time", "Energy", "Inertia" [5a], and, more concretely 
- on the outstanding von Weizsäcker's 1953-54 year "Ur-hypothesis" [20, 21] that Matter on fundamental depth is based on a binary logic, and just therefore Matter's space is 3-dimensional, and

- on outstanding Fredkin-Toffli's finding [22], who showed that if the elements in a system are based on a reversible logic, the system changes at the elements interactions without energy dissipation outside the system.

Correspondingly in the informational physical model it is rationally so postulated that Matter is based on binary reversible logics, which is actualized as the Matter's "ether" [5]4D dense lattice of binary reversible fundamental logical elements (FLE), which [lattice] is placed in the Matter's fundamentally absolute [5]4D "Cartesian" spacetime an empty container with the metrics $(c \tau, X, Y, Z, c t)$, where Matter exists and constantly changes,

- and where $X, Y, Z$ are 3 "von Weizsäcker's" space dimensions, which correspond to "ordinary" binary degreases of freedom at FLE state changes - "FLE flips",

- "ct" is the "true time" dimension, and

- " $c \tau$ " is the "coordinate time" dimension, which really is a specific space dimension as well, and is necessary to implement the FLE degree of freedom of reverse sequences of changes, which are in a sense "non-legitimate" in the true time (i.e. the universal Set's time dimension), as some "travels backward in time".

Note, that in this case for some reasons, first of all as actualization of experimentally observed in this case "equal footing" in the space and the times dimensions, in the metrics above instead of " $t$ " for the true time, and " $\tau$ " for the "coordinate time" dimensions the " $c t$ " and " $c \tau$ " units are used, $c$ is the standard speed of light.

\section{2 "What is "a particle""}

From the above, and from experimental fact that the particles, in spite of that constantly change, are, nonetheless stable, in the model it is quite rationally postulated that particles in Matter are some cyclic close-loop algorithms, which constantly run as cyclic sequential disturbances in the FLE-lattice, ad so they always move in the 4D subspacetime with metrics ( $c \tau, X, Y, Z)$ with the 4D speeds of light, $\vec{c}$, and, in parallel, in the $c t$-dimension with the speed of light; the absolute value of which is determined by FLE lattice parameters - by FLE "size", which is assumed be equal to Planck length, $l_{P}$ and FLE "flip time", Planck time, which is assumed be equal to $t_{P}$, so $c=l_{P} / t_{P}$.

Rather essential evidence that these assumptions are adequate to the reality reveals itself at comparison of Gravity and E/EM forces strengths in [5a]

Particles are created if some FLE in the [5]4D FLE lattice is impacted with transmission to this FLE some 4D momentum,

- that cyclically change their internal states with frequency $\omega$ so that a particle has energy $E=\hbar \omega=m c^{2}, m$ is the inertial mass, $\hbar$ is the fundamental elementary physical action, reduced Planck constant, $c$ is the speed of light. This hypothesis appeared as early as in 1920 as the "the Zitterbewegung". de Broglie hypothesis [26], [27]. 
A few naturally suggested, and postulated in the informational model, rational premises follow from that above:

(i) - particles are some cyclic disturbances of the FLE lattice, which appear when a 4D momentum impacts on an ether FLE, which, after the impact, "flips" further causing sequential flipping of neighbor FLEs.

To cause a flip - and the corresponding sequential flipping of ether FLEs along a straight line an infinitesimal momentum is enough, however if the momentum isn't infinitesimal, since the FLE flipping point cannot propagate with a speed that is larger than $c$, the direct line transforms into a 4D "helical" the FLE-flip point motion of correspondingly close-loop precessing sequentially flipping FLEs - and a particle appears. The flip point at that moves along the 4D "helix" with the speed $c \sqrt{2}$, i.e. it seems that the FLE flipping happens "diagonally", nonetheless the "helix front" moves along the impacting 4D momentum direction with 4D speed of light, $\vec{c}$.

(ii) From the existing experimental data it seems rational to assume (in first approximation, see point (i) above) that the "radius" of the "helix" is equal to the particle's Compton length $\lambda=\frac{\hbar}{m c}$,

(iii) The always moving particles are, thus, some "gyroscopes" which are always oriented relating to the propagating direction, and

(iv) Note also, that it follows from the experimental data that there are two main types of particles in Matter, depending on the parental 4D momentums. In the model that are "S-particles", created by spatial momentums, and "T-particles", created by momentums that were directed in the "coordinate time", i.e. along the $c \tau$-axis. So photons are Sparticles, whereas most of other particles are T-particles, which so can be at rest in the $3 \mathrm{D}$ space, moving along the $c \tau$-axis with the speed of light. Motion of T-particles in the space appears after some space-directed momentum impact, at that T-particles inertia reveals itself as the particles" "rest masses".

From the points (i) - (iv) above the Lorentz transformations and the rest in fast bodies mechanics follows.

Besides, in the model in complete consistence with existent experimental data it is supposed - and postulated - that antiparticles, are, as the particles above, also some close-loop algorithms, which are the same as corresponding particles' algorithms, but the algorithms run in reverse command order, and

- in the model the problem "what are fundamental Nature forces" is rather possibly rationally clarified: the Nature Forces are some marks on the particles algorithms, which act causing special disturbances in the FLE lattice, which are marked by a Force, i.e. "Forces mediators", which transmit, if hit, to other particle's algorithm that has the same mark, some momentum. Three Forces (Weak, E/M, and Strong Forces) make so particles algorithms asymmetrical, whereas only Gravity Force is symmetrical - in Matter gravitationally everything equally attracts everything.

The binary logics is actualized, and experimentally observed, in Matter as that all objects/events/processes are linked by the couple of "active" - unlike the "passive" 
Planck parameters the FLE size and the FLE flip time - the fundamental elementary physical action, $\hbar$, and the fundamental elementary angular momentum, which also is equal to $\hbar$. This equality rather probably isn't random, however now this point isn't clarified in this model state, however note, that at elementary changes of a spin states of QM objects on $1 \hbar$ is the change of information on 1 bit, and it looks as well rational that that happens also at changes of physical action of the objects. Thus the least action principle really states that real changing in states of material objects happens by the condition of minimum binary operations/minimal informational change.

From the above rather rationally it follows, that the inequalities in the Heisenberg uncertainty principle really are equalities.

\section{A couple of additional to [5a] fundamental problems}

\section{1"Antiparticeles and QM"}

Note, that the answer on this question is possible only if this problem is considered again in the absolute [5]4D Euclidian spacetime metrics $(c \tau, X, Y, Z, c t)$, where particles are always constantly moving with 4D speed of light in the 4D sub-spacetime with metrics $(c \tau, X, Y, Z)$ having the 4D momentums $\vec{P}=\left(p_{c \tau}, p_{X}, p_{Y}, p_{Z}\right), \vec{P}=m \vec{c}$ (and particles' energies $\left.E=P c=m c^{2}\right), P^{2}=\sum_{j=1}^{4} p_{j}^{2}$; and, simultaneously, are moving in parallel, in 1D ct-dimension with the speed of light and the momentum $\vec{P}=m \vec{c}$.

As that is pointed above antiparticles, are, as the particles above, also some close-loop algorithms, which are the same as corresponding particles' algorithms, but the algorithms run in reverse command order. For S-particles that isn't too essential, and so photons haven't essentially different antiparticles, and differ only by the \pm helicity, however T-particles and T-antiparticles differ essentially, since the $c \tau$-dimension is specific one, and so these particles have opposite momentums in the in this dimension, $p_{c \tau}($ particle $)=-p_{c \tau}$ (antiparticle).

In contrast, since in Minkowski space the dimension/variable " $t$ " is really some mix of the true time, $t$, and coordinate time, $\tau$, so "4-momentums" in SRT, $\vec{P}_{M}$, physically are rather strange: $\vec{P}_{M}=\left(\frac{E}{c}, p_{X}, p_{Y}, p_{Z}\right)$ i.e. the zero component of a 4-momentum of a particle is, in fact, the whole real 4D momentum - and the momentum in true time, whereas really the " $t$ " in Minkowski space has also the coordinate time traits, as it is the "proper time" of, a moving in a "stationary" frame, frame. Besides that is "the time, which clocks read", which (clocks) are usually some T-bodies, and really show how they move in the coordinate time dimension

Though, of course, in a rigid reference frame really it is impossible to observe motion in the true time.

So in Minkowski space - as that really is fundamentally in true time - in the time dimension there is no backward in time motion, and antiparticles do not exist in SRT. 
Correspondingly, when Dirac developed in framework of SRT QM equation for fast moving free particle - electron, he made that as modification of existent already nonrelativistic Schrödinger time dependent equation $i \hbar \frac{\partial}{\partial t} \psi(\mathbf{r}, t)=\hat{H} \psi(\mathbf{r}, t)$, where $\psi$ is the wave function of a particle, $\hat{H}=\sum_{j=1}^{3} \frac{\hat{p}_{j}^{2}}{2 m}$ is the Hamiltonian operator, which corresponds to the classical mechanics Hamiltonian $H=\sum_{j=1}^{3} \frac{p_{j}^{2}}{2 m}, \hat{p}_{j}=-i \hbar \frac{\partial}{\partial x_{j}}$, $j=1,2,3$, are [really partial] operators of 3D space particle's momentums;

- and using, instead of classical Hamiltonian above, the relativistic Hamiltonian $H=c \sqrt{p^{2}+m^{2} c^{2}}$, where $p$ is the absolute value of $3 \mathrm{D}$ momentum, $p^{2}=\sum_{j=1}^{3} p_{j}^{2} m$ is rest mass of a particle, $c$ is the speed of light.

This Hamiltonian has the 3D space momentums in the square root, and so it is impossible to obtain directly linear differential equation for the wave function using the momentums operators above - as that is made in the Schrödinger equation. However Dirac solved this problem by beautiful non-standard way; in which, besides, the Pauli spin formalism was taken into account quite naturally, wave function of particles turns out to be 4-component vector that describes particle's complex quantum state - what looks as indeed adequate to the reality, etc. So in the equation the "Dirac Hamiltonian" is $\hat{H}=m c^{2} \alpha_{0}+c \sum_{j=1}^{3} \alpha_{j} \hat{p}_{j}$, where partial momentum operators are the same as in Schrödinger equation above, $\alpha_{0}$ and $\alpha_{j}$ are the four $4 \times 4$ matrices, which determine the four-component - in contrast to the one-component $\psi$-function in Schrödinger equation, $\psi$-function. The equation is in complete consistence with the SRT formalism, including that happens, if in this Hamiltonian $m c^{2}$ has negative value what formally is possible because of, as that Dirac told, e.g., on the first "Atomic nucleus" conference in the USSR [34] (translated from Russian):

“....In Newton mechanics kinematical energy $W$ is always positive. But in Einstein theory. $W$ is defined by more complex equation, namely

from what

$$
W^{2}=m^{2} c^{4}+p^{2} c^{2}
$$

$$
W= \pm \sqrt{m^{2} c^{4}+p^{2} c^{2}}
$$

In classical theory from both signs always + is chosen, what does with purpose to obtain accordance of the theory with experiment.

That creates no difficulties, since from our equations is seen, that $W$ can be only more than $+m c^{2}$, or lesser than $-m c^{2}$. That connotes that the states with positive kinetic energy are separated from the states with negative kinetic energy by the interval $2 m c^{2}$ (from $+m c^{2}$ to $-m c^{2}$ ), and, since in classical theory all dynamical variables are continuous, so a particle, which had firstly a positive kinetic energy, by no means can To transit into a state with negative energy. By different way that is in quantum theory: the wave equation has the property that existence of some disturbing force obligatorily creates for the particle a probability of transition from initial state with positive kinetic energy into a state with negative 
kinetic energy:: therefore in quantum theory it is impossible simply to ignore a possibility of negative kinetic energy, as that is in classical theory ..."

Finally, the obtained equation is

$$
i \hbar \frac{\partial \psi(x, t)}{\partial t}=\left(\alpha_{0} m c^{2}+c \sum_{n=1}^{3} \alpha_{n} p_{n}\right) \psi(x, t)
$$

where the Dirac's approach above is introduced as "hole theory", that the vacuum is the many-body quantum state in which all the negative-energy electron eigenstates are occupied. This description of the vacuum as a "sea" of electrons is called the Dirac sea.

The approach looks as rather questionable, since "negative-energy states" for free particles really don't exist.

However if Dirac would consider this problem as the "momentum problem", he could consider the equation for the momentum $m_{0} \vec{c}= \pm\left(\frac{W^{2}}{c^{2}}-p^{2}\right)^{1 / 2}$

- where the sign " \pm " for the vector momentum $m_{0} c$ is undoubtedly legitimate unlike to scalar energy $W$ above, and so the hypothesis about the particles that move with negative speed of light - oppositely to electrons, would be lesser questionable as well.

And really the Dirac equation is practically the equation for the momentum, if both sides are divided by $c$

$$
i \hbar \frac{\partial \psi(x, t)}{\partial(c t)}=\left(\alpha_{0} m c+\sum_{n=1}^{3} \alpha_{n} p_{n}\right) \psi(x, t)
$$

- however that would be, nonetheless, again impossible in framework of the SRT, where such momentums don't exist.

Really - see above - the T-particles electrons, and positrons, are created by oppositely directed in the $c \tau$-axis momentums, and that above is, besides, the answer on the problem

\section{2 "What is the "Feynman-Stueckelberg interpretation" in QED [35], [36], where it is postulated that antiparticles move backward in time"}

The motion with negative speed in the $c \tau$-dimension practically for sure happens in Matter, if, as that is again rationally suggested and postulated in the informational model, the antiparticles have the same algorithms as the corresponding particles, but their algorithms run in reverse command order, therefore

- the antiparticles really move backward, however not in the true time, but backward in the coordinate time, which is just "the time what clocks read", and which really is measured as experimental base of physical theories. It also matters that existent clocks are made from particles, and so real positions and motion of antiparticles on the $c \tau$-axis are experimentally non-observable. If it would be a possibility to make a clock from antiparticles, that would be possible - as observation that on such clock the pointer rotates oppositely to the pointer on its made from particles twin. 
Note, though, also - the coordinate time is not the time; and so, for example, if there would be two twins, one "material" and the other "antimaterial", their clocks would show opposite signs of their ages. However, both twins will seem practically identical as usual twins, since biologically they would age principally only in the true time. Not completely, though - moving in the space both twins would biologically age slower - in the coordinate time, than the twins at absolute rest.

\section{Cosmology}

There are many problems in cosmology, first of all, in some cases of principally insurmountable uncertainty even in the formulation of these problems, since this physical branch often relates to objects, events, and processes which humans in controlled conditions cannot study now.

However, the informational physical model allows to consider a few problems rationally enough. These problems can not be principally rationally solved or clarified in mainstream physics, i.e. outside the "Information as Absolute" concept and the model.

\subsection{The "Beginning problem"}

This is an utmost fundamental problem in cosmology, and it is rather evidently principally irresolvable in framework of official physics. Physics has no reliable data about the objects, events and processes that could exist, appear, and happen at Beginning. Nonetheless a number of theories exist in physics, and in the standard cosmological "Big Bang" model [40] it is suggested concretely that

“....As the Big Bang theory goes, somewhere around 13.8 billion years ago the universe exploded into being, as an infinitely small, compact fireball of matter that cooled as it expanded, triggering reactions that cooked up the first stars and galaxies, and all the forms of matter that we see (and are) today....."

- in spite of that the existent physics principally is not applicable to this "infinitely small, compact fireball of matter", etc.

As well as to the next steps of Matter's creation, when in the model

"...more explosive phase of the early universe at play: cosmic inflation, which lasted less than a trillionth of a second. During this period, matter - a cold, homogeneous goop — inflated exponentially quickly before processes of the Big Bang took over to more slowly expand and diversify the infant universe......"

- existent physics knows absolutely nothing about what was this "cold, homogeneous goop"; why "it inflated exponentially quickly before next processes of the Big Bang", by what reason this "inflation" stopped; and further by what reason and how that "took over to more slowly expand and diversify the infant universe", etc.

Nonetheless, there exist, basing on existent astrophysical data, a number of seems as rather rational points in standard model of Matter's evolution after Beginning, including, if we do not take into attention the remark above, the rather rational "phenomenological" description of states in Matter evolution above,

- starting from the "space inflation" state/epoch [41], [42], when the space, in the standard model for unknown reasons, and by some transcendent way, exponentially 
expanded, and that happened at some relaxation of some completely unknown in physics "inflaton" field's singularity, because of "a repulsive gravitational force" (?) [40]. However, the "inflation hypothesis", in spite of these rather questionable points, seems adequately to the reality phenomenologically describes the observed uniformity of matter density and of the material objects nomenclature on cosmological distances, the nucleosynthesis, etc.

Including the hypothesis in the standard model that during inflation the matter was a cold, homogeneous goop, seems is rather plausible, since that is consistent with cosmological observations. However, that contradicts with the assertion that the matter "exploded into being, as an infinitely small, compact fireball" in this model in the quote above.

The informational approach allows to formulate reasonable physical hypothesis [5], [7] in accordance with the existent experimental data and with reasonable points in the standard Big Bang model above, such as the inflation epoch, and that the Matter after the inflation was rather cold, etc.

In the hypothesis it is suggested that the "Information" Set's element "informational system "Matter"" was created by the other Set's element, "an informational system conscious smart "Creator"', which was indeed extremely smart and could design a logically simple, however functionally extremely complex, effective, and closed in the Set, informational system; and find at creation of this system a few huge portions of the mysterious for humans till now phenomenon "Energy" in the Set.

Thus - see above - Matter is based on the simplest binary and reversible logics + (at least) 4 fundamental logical marks, which humans observe as 4 real fundamental Nature forces, including Gravity, and few universal links and constants, which are "written" in the Matter's utmost fundamental base - in the correspondingly binary [5]4D reversible fundamental logical elements [FLE].

Further this design was actualized into Matter in the next 3 steps - and portions of energy:

On the first step the [5]4D dense lattice of [5]4D FLE was created ("inflation epoch") exponentially, as the result of programmed division, possibly into 2, of possibly one "primary FLE" (as that, say, bacteria spread in a Petri dish, if there are enough resources) in the corresponding Matter's absolute [5]4D spacetime with metrics $(c \tau, X, Y, Z, c t)$, Euclidian of course,

- which "automatically", i.e. by definition of the absolutely fundamental phenomena "Space" and "Time" - see section 2.4.6 above, appeared at the creation yet of the "primary FLE". Note, though, that this spacetime always existed in the Set, which exists absolutely fundamentally always, i.e. without Beginning and End, as a sub-spacetime of the Set's whole spacetime. The FLE lattice was cold;

- on the second step, the energy portion with $c \tau$-directed momentums was globally uniformly pumped in this FLE-lattice, and there the completely symmetrical primary Tparticles were globally uniformly created. It seems as rather probable that the energy was spent only on the particles creation, and so the matter in Matter was probably rather cold again. 
However from existent cosmological data it looks as rationally to assume that the pumping wasn't uniform locally - in the lattice [and so in 3D space] some clusters of primary particles were created, where the particles density was radically enhanced, which were some seeds of appearing on next Matter's evolution steps large cosmological objects, first of all - galaxies.

At that the next fundamental problem, which exists since reality evidently contradicts with existent experimental data in high energy physics, and corresponding physical theories

\section{2 "Why Matter now practically does not contain antimatter",}

seems to be well rationally solved:

- Matter does not contain antimatter since it did not contain antimatter yet at the second step, because the primary T-particles were completely symmetrical algorithms. In this case it is illogical to consider the difference "particle/antiparticle". Hence, it was logically completely permissible for all primary particles to be only "particles" - i.e. which have the positive momentums in the $c \tau$-dimension, and

- on the third step the primary particles (which in the hypothesis are rather probably Planck mass particles or other simple particles, i.e. that were symmetric algorithms and have only completely symmetrical gravitational charges) interacted by using only completely symmetrical Gravity force, the result was, rather possibly indeed a soup of only "ordinary" particles, which was distributed again globally uniformly - but nonuniformly locally - in the lattice.

In the "soup" unstable particles decayed quickly and - as the standard cosmology asserts rather adequate to the reality - the observable now particles eventually remained, and this soup was rather hot. Hence, CMB exists now, however that possibly was not a "singular" temperature, because the energy was mostly spend on creation of the ordinary particles.

If the primary particles were the Planck mass particles, then nearly $10^{19}$ "ordinary" baryons were created in an interaction of two particles.

At that Creator practically for sure did not need to control the step-2 and step-3. Creator well knew that nothing besides a concrete informational system "Matter" can appear, if a dull energy is pumped in the FLE-lattice; and, say, this Matter could have a number of thousands of galaxies lesser or more, but for Creator that was not essential.

The hypothesis presents a rather rational answer on the fundamental physical problem

\section{3. "What is the "dark matter""}

- it would not be surprising if, say, the "dark matter" indeed exists, made up from the "primary particles". That could happen if during the creation of "ordinary" matter only $10-30 \%$ of these particles have interacted, and $70-90 \%$ of the "relics" exist till now. If these are the Planck mass particles, then the density of the dark matter particles is in $10^{19}$ times lesser than the baryons' density, i.e. 3-4 particles in a cube with the size 1000 $000 \mathrm{~m}$. 
Since the primary particles interact only gravitationally, they interact with "usual" particles at a probability extremely lesser than when that for neutrinos, and so (i) - the bodies, stars, etc., are practically transparent for these particles, which rotate around centers of some massive bodies along their single own orbits, forming corresponding haloes, and (ii) - they are practically non-detectable, due both to extremely small cross section and extremely small concentration.

Though if an interaction happens in a detector, that will be well observable, $10^{19} \mathrm{BeV}$ is rather observable energy.

Besides it looks as rather rationally to assume, that in the "seeds" clusters there were some local - and small - regions, where the primary particles density was so large, that the primary particles composed compact objects with extreme mass and Gravity field, which have became centers of galaxies, having masses millions, even billions, of stars.

These objects have some interesting physical trait - the strength of created by the objects Gravity field is so large, that escape velocity becomes be equal to the speed of light, and so, say, even photons, if aren't radiated orthogonal to the objects surface, propagate inside corresponding space volume along closed orbits - such objects so practically don't radiate light.

That happens in both existent theories of Gravity - Newton's theory and general relativity, and happens at least provided that the mass, $M$, and radius, $R$, of an such object are in accordance with the equation $R_{g S}=\frac{2 G M}{c^{2}}, R_{g S}$ is the radius in GR (Schwarzschild radius), corresponding radius, $R_{g N}$, in Newton Gravity is two times lesser. At that the radius isn't the object's radius, really it can be lesser than that radiuses above.

The difference of $R_{g S}$ and $R_{g N}$ values isn't principal, however these radiuses principally differ in that when $R_{g N}$ is the radius of some "virtual" surface, which surround some "dark place", the Schwarzschild radius is the radius of the "event horizon" in GR, where solutions of the GR equations become to be singular, and so the event horizon is the border of a "hole in spacetime" - a "black hole" $(\mathrm{BH})$, and so nothing principally can escape from this hole.

Really on the event horizon no singularity exists, the potential and strength of Gravity field increase rather smoothly with decreasing of the distance to the center of the object. So, say, the super massive black holes (SMBH) in centers of galaxies, which, rather probably, are offspring of the "seed" objects that were growing absorbing gas and other matter around at galaxies' evolutions, have rather large Schwarzschild radiuses, whereas the sizes of compact objects in SMBH evidently are mach lesser than the radiuses.

For example Sagittarius A* (SMBH in Milky Way) has mass, $M=8.2 \times 10^{36} \mathrm{~kg}$, and corresponding event horizon radius $R_{g S}=1.2 \times 10^{10} \mathrm{~m}$. So average density of matter in this SMBH, $\rho=1.1 \times 10^{6} \mathrm{~kg} / \mathrm{m}^{3}$. This density is much lesser than the density of neutron stars' matter $10^{17}-10^{18} \mathrm{~kg} / \mathrm{m}^{3}$, and so even if in Sagittarius A* center some big neutron star would be placed, its radius would be $\sim 10^{4}$ times lesser than the Sagittarius A* "event horizon". 
Thus it looks as rather rational to assume, that in this case the phase of SMBH matter state is the next phase after known now phases "ordinary matter", "white dwarf" and "neutron star" matter, and rather probably the object is some dense composition mostly of the primary particles, whereas the space between the object's surface and "event horizon" is filled by the accretion disk continuance, which is practically unobservable, and by some other particles that have diffuse distribution.

And it looks as rather probable to propose that there don't exist some sharp border for matter in the event horizon, in the mechanics existence of "escape velocity" for some body by no means determines some limits of distances that lesser bodies in the "body's atmosphere" can move on which, if lesser body speeds value is near the escape velocity value, the lesser body can move on practically infinite distance. It looks as rational to suggest, that that is true in the case when the escape velocity is equal to the speed of light as well.

If that is so, then really a "black hole", besides the accretion disk, has practically for sure, some "atmosphere". Including in the observed SMBHs' jets, including the "bubbles" of Sagittarius A* SMBH it is nothing surprising - that are, rather probably, some SMBH "atmosphere's" specific details, which, as that is explained in standard cosmology, are formed by magnetic fields that are formed by the accretion disk and SMBH itself.

However, it looks as rather probable, that this explanation at least possibly isn't complete, for example the Earth radiation belts that are formed by Earth magnetic field, aren't some jets, moreover, the charged particles in the belts move mostly toward Earth in polar regions - oppositely to the jets.

Though, of course, the magnetic field of some SMBH isn't formed like that the Earth's "dynamo" does, much more probably the field can be formed by rather probable the huge "neutron star phase matter" skin on the SMBH compact object above, and so the SMBH jets are formed as that happens in the "ordinary" observable pulsars,

- however we cannot exclude that the jets are formed (including in the pulsars) by some other than magnetic force ways also, for example - by gravimagnetic force. To consider this case, though, we should have real theory of Gravity, which doesn't exist till now, however it looks as rather rational to assume, that this effect can exist, and can be observable if an object is large enough, for example that possibly could be Sun.

Correspondingly it looks as probable that at measurements of the spatial distributions of concentrations of ions, neutral particles, other matter, around Sun in the cosmic missions on polar orbits around Sun, the "SMBH features", i.e. some accretion disk and jets/bubbles can be observed; and, because of that will be made in rather controlled conditions, the measurements' results in this case would be useful in both cases - at development of SMBH physics, and at development of the real Gravity theory. As well as on the missions some other instruments could be installed, for cosmology studying of some concrete star looks as more useful than, say, studying of planets in the Sun system and seeking for planets in other stars' systems.

\section{4 "What is the "dark energy""}

Both interpretations of existent cosmological data as "space expansions", i.e. the exponential "inflation" on the first step of Creation, and more tolerant next one that 
rather probably really proceeds till now, really, if happen, are the FLE lattice expansions; and to make that it was - and is - indeed necessary to pump into the lattice essential energy. However, this energy is completely outside physics, and so attempts to incorporate this energy in existent physical theories, as that is in cosmology as the introducing of Lambda term in the GR equations, really are irrational.

Though, as that is in the Beginning model above, this [in the model - Creator's] energy can be used in rational descriptions of what and how happened in first instants at Beginning, or, for example, when for description of the FLE lattice expansion after appearance of "ordinary" Matter it looks as would be possible to find some rational reasons as well. For example, this expansion would be necessary to prevent Matter's collapse because of the "gravity paradox" [43]; though here can be many other reasons, of course.

\section{Conclusion}

This paper, in fact, is some "conclusion" for the existent now series of papers, where the "The information as Absolute" concept, the informational physical model, and concrete physical problems in framework of the concept and the model, are considered. Hence, a typical conclusion would be too long for this paper.

So here only a few final remarks that relate to possible development of the model and its application in physics.

Firstly, more rational versions of traditional physical theories should be developed. The current theories are mostly based on the SRT formalism, first of all, on the postulate that real Matter's spacetime is the 4D Minkowski space, and so the phenomena "Space" and "Time" are actualized in the theories really erroneously.

Instead physics should be re-formulated in accordance with the fact that real Matter's spacetime is the absolute [5]4D Euclidian spacetime with the metrics (cc,X,Y,Z,ct), where time doesn't flow somewhere. However, in everyday physical practice rather probably the passed way of a body formula will be as it is now, $S=V t$, where the time " $t$ " " of itself, and from its own nature flows equably".

Correspondingly in this case it is necessary to develop the theory of the 4D angular momentum, Hamilton and Lagrange functions, etc.; note also that in this case the least physical action principle looks as is based more physically.

Correspondingly, after the mechanics reformulation, it will be necessary to re-formulate corresponding QM operators, including in this case a next fundamental physical problem

\section{"Why time in QM does not have a corresponding operator"}

- would be solved as well.

This problem, though, appears also because of other fundamental problem - that the time-dependent Schrödinger and Dirac equations are fundamentally - and so really essentially - incorrect, since are as $i \hbar \frac{\partial \psi}{\partial t}=\hat{H} \psi$, whereas, at that, in QM the 
derivations by other than time observables/variables $\hat{p}_{j}=-i \hbar \frac{\partial}{\partial x_{j}}, j=1,2,3$, are operators of 3D momentum. It looks as quite natural, that the derivative by the observable "time" is also the momentum operator, and so the equation for wave function really should, rather probably, be as $i \hbar \frac{d \psi}{d(c t)}=\hat{P} \psi$; where the whole momentum operator $\hat{P}$ is composed from the partial operators $\hat{p}_{j}=-i \hbar \frac{\partial}{\partial x_{j}}, j=1,2,3,4$; though taking into account that these operators aren't independent, since $P^{2}=\sum_{j=1}^{4} p_{j}^{2}$.

In this case the observable "time", more correctly - both, true and coordinate times, observables, become to be "ordinary" observables, and so have the operators themselves, as that 3 space observables are now in QM.

However, that isn't only QM problem - this problem has the root in the Hamiltonian and Lagrange formalisms in classical mechanics, where the variable time also essentially differs from the spatial variables; first of all, because of the Newton-SRT definition of time (see the section 2.4.6 above); and the re-formulation of classical and SRT mechanics that is pointed above can be essential for the QM re-formulation.

Returning to the other QM problems note also that at considering of QM events and processes in absolute frames, when some QM objects are free, the problem of causality, which occur in some cases in standard physics, doesn't appear, since in this frame all clocks show real positions of objects in the true and coordinate times, which (positions) have in this case the same values.

Besides, a re-formulation of QM, if would take into account the really existent [5]4D FLE ether, possibly will result in better understanding of the QM phenomena; including, possibly, of really existent fundamental problem "What is the Pauli principle, and is or not the force that limits number and spins of fermions in a given state a "fifth fundamental Nature force" or that is something else?"

Note, though, that introducing the FLE approach in Standard Particles Model and QFT seems will be more fruitful, first of all in the last case - at solution of the "virtual particles" problem.

In Standard Model, the version of CPT theorem allows to obtain rather questionable results, such as the solutions [44], [45] of the section 5.2. problem, where at Beginning both "Matter" and "AntiMatter" appeared; and, in accordance with the CPT theorem, they then immediately turned out to be divided in "spacetime" and in "antispacetime" (?); just so Matter does not contain antimatter now. Thus the CPT theorem must be reformulated as well.

Besides it seems rational to suppose that the popular in the mainstream physics problem of "development of the "[Grand] Theory of Everything" which will "unite" all existent fundamental Nature forces, really is not actual. Really Forces are functionally fundamentally different, and so rather possibly really here is no any necessity in some "unifications". 
Though some "unifications effects" can appear, mostly at exotic energies, when problems with sufficiency of numbers of FLEs in some particles algorithms' to mark all the particles' charges (which the particles have not in more tolerant conditions), can appear at some interactions of the particles; and corresponding observed effects can be used at development of Standard Model.

However utmost important problems now are (i) - the problem of "virtual" particles and interactions in existent quantum dynamics theories, which should be replaced by real ones; for QED and QGD rather possibly that will be made basing on the FLE approach and taking into account the initial models of Gravity and Electricity (Sec.4.3 above); that is true for classical ED and GD, of course; and (ii) what relates to all classical, quantum, and for the initial models - problem of what is the energy/momentums that the Forces transmit to particles at mediators' impacts?

Etc., more see the main text.

Finally note that the experiments, which are proposed in the informational model, i.e.:

(1) - the observation of the absolute motion and measurement of the absolute velocity of the peculiar motion of the Solar system [10], [11]; though the measured absolute velocity will be the same as which follows from the CMB dipole measurement with a rather non-zero probability, because from the model of Matter's creation in section. 5 it follows that Sun - as any other object in Matter's space - is in essentially a cold the spacetime region and so hasn't some exotic absolute speed. Note, though, that there exist other estimations of the velocity at observations of quasars, distant AGNs or $\mathrm{SNe}$ Ia, which results in the velocity values that are essentially larger than the CMB value [46]; and so this experiment's results would be useful at solution of this puzzle;

(2) - the observation of the quantum nature of Gravity [3], [8], and

(3) - the important, simple and cheap, though not really fundamental, experiment [12] (viXra file version), which, rather probably, will show that the GR postulate that photons at motion do not change their energy in gravitational fields between points that have different potentials, is wrong;

(4) - measurement of distributions of density and momentums of charged and neutral low energy particles and matter around Sun by instruments on polar orbits (section 5.3);

- should be made as soon as possible.

\section{References}

[1] S.V. Shevchenko, V.V Tokarevsky, "The Information and the Matter." e-print arXiv: physics/0703043v5 (2007-2008)

[2] S.V. Shevchenko, V.V Tokarevsky, "Inform Physics do is possible?" Poster report on the conference XIXèmes Rencontres de Blois Matter and Energy in the Universe. Blois, Loire Valley, France May 20th - May 26th, (2007)

[3] S.V. Shevchenko, V.V Tokarevsky, "The information as Absolute" e-prints arXiv:1004.3712v2, http://viXra.org/abs/1402.0173, 
https://www.researchgate.net/publication/260930711 the Information_as_Absolute http://dx.doi.org/10.5281/zenodo.268904 (2010-2017)

[4] S.V. Shevchenko, V.V Tokarevsky, "The informational physics indeed can help to understand Nature?" e-print http://arxiv.org/abs/0812.2819 (2008-2010)

[5] S.V. Shevchenko, V.V Tokarevsky, "The Informational Conception and Basic Physics" eprint arXiv:0707.4657v4 , http://viXra.org/abs/1503.0077

,https://www.researchgate.net/publication/273777630 The_Informational_Conception_and_Bas ic_Physics http://dx.doi.org/10.5281/zenodo.16494 (2012-2015)

[5a] S.V. Shevchenko, V.V Tokarevsky, v1-v4 "The Informational Conception and Basic Physics" e-print arXiv:0707.4657v5 (2021)

[6] S.V. Shevchenko, V.V Tokarevsky, "The informational model - possible tests" e-print http://arxiv.org/abs/0706.3979 (2011)

[7] S.V. Shevchenko, V.V Tokarevsky, "Space and Time", e-print http://arxiv.org/abs/1110.0003 (2013)

[8] S.V. Shevchenko, V.V Tokarevsky "To measure the absolute speed is possible?", e-print http://viXra.org/abs/1311.0190

https://www.researchgate.net/publication/259463954 To measure the absolute speed is poss ible DOI 10.5281/zenodo.34960 (2013)

[9] S.V. Shevchenko, V.V Tokarevsky, "Measurement of the absolute speed is possible?", eprint , http://vixra.org/pdf/1311.0190v4.pdf http://dx.doi.org/10.5281/zenodo.48709 (2016)

[10] S.V. Shevchenko, V.V Tokarevsky, "The informational model - gravity", e-print http://vixra.org/abs/1409.0031 http://dx.doi.org/10.13140/2.1.4332.9925 (2016)

[11] S.V. Shevchenko, V.V Tokarevsky, "The notion "speed" and the Lorentz transformations", e-print

https://www.researchgate.net/publication/317067896_The notion_speed_and the LLorentz tran sformations http://dx.doi.org/10.5281/zenodo.802365 (2017)

[12] S.V. Shevchenko, V.V Tokarevsky, "About some conventions in mechanics", e-print https://www.researchgate.net/publication/317620440 About some conventions in mechanics , http://vixra.org/abs/1712.0673 http://dx.doi.org/10.5281/zenodo.1142628 (2017)

[13] S.V. Shevchenko, V.V Tokarevsky, "The informational model: twin paradox", e-print https://www.researchgate.net/publication/322798185 The informational_model twin_paradox http://dx.doi.org/10.13140/RG.2.2.34064.51201/1

[14] S.V. Shevchenko, V.V Tokarevsky, "The "Information as Absolute" conception: the consciousness", e-print https://www.researchgate.net/publication/329539892 The Information_as_Absolute_conceptio n the consciousness , http://viXra.org/abs/1812.0406 DOI:

http://dx.doi.org/10.13140/RG.2.2.26091.18720/1 (2018)

[15] S.V. Shevchenko, V.V Tokarevsky, "The Information as Absolute" conception: Marxism and "now", e-print

https://www.researchgate.net/publication/321757886 The Information as Absolute conceptio

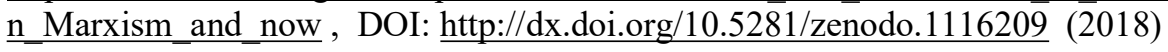


[16] M.J. Duff, “Top ten problems in fundamental physics" International Journal of Modern Physics A Vol. 16, No. 05, pp. 1012-1013 (2001) special issue: strings 2000

[17] E. A. Roland and S. Lidström, "Life, the universe, and everything - 42 fundamental

Questions" e-print arXiv:1804.08730v1 [physics.pop-ph] (2018)

[18] I, Newton, Philosophiae Naturalis Principia Mathematica. (1686). in: Newton's Principia : the mathematical principles of natural philosophy https://archive.org/stream/newtonspmathema00newtrich\#page/n349/mode/2up

[19] A. S. Eddington, "The Nature of physical World" Cambridge at the university press (1948)

[20] C.F. von Weizsäcker, "Eine Frage Über die Rolle der quadratischen Metrik in der Physik Zeitschrift für Naturforschung", 7 a: 141, (1952).

[21] C.F. von Weizsäcker, "Komplementarität und Logik. Die Naturwissenschaften”, 42: 521$529,545-555,(1955$.

[22] E.Fredkin, "Digital Philosophy",

http://www.digitalphilosophy.org/digital_philosophy/toc.htm (2000)

[23] H. Poincaré, "Science and Hypothesis", (New York: the Walter Scott publ. p. 171,(1905)

[24] A. Einstein, “Zur Elektrodynamik bewegter Körper”. Ann. Phys., 322: 891-921. (1905),

[25] H. Dingle, "The Case against Special Relativity". Nature. 216 (5111): 119. (1967)

[26] L. De Broglie, "Recherches sur la th'eorie des quanta, R'eedition du texte" (1924), Masson \&Cie, Paris (1963)

[27] M. M Asif, and.S. K. "Zitterbewegung, internal momentum and spin of the circular travelling wave electromagnetic electron" e-print arXiv:1602.07534 [physics.gen-ph] (2016)

[28] G. F. FitzGerald, "The Ether and the Earth's Atmosphere" Science, 13 (1889)

[29] H . Minkowski, "Space and Time”, in. Lorentz, Hendrik A., Albert Einstein, Hermann Minkowski, and HermannWeyl, "The Principle of Relativity: A Collection of Original Memoirs on the Special and General Theory of Relativity", Dover, New York (1952)

[30] H. A. Lorentz, "Electromagnetic phenomena in a system moving with any velocity smaller than that of light", Proceedings of the Royal Netherlands Academy of Arts and Sciences, 6: 809-831 (1904)

[31] H. Poincaré, “Sur la dynamique del'électron”, Comptes Rendues, V 140, p1504 (1905)

[32] H. Poincaré, "Sur la dynamique de l' electron", Jourmal Rendiconti del circole Matematico di Palermo; (1906)

[33] J.S. Bell, "How to teach special relativity", Progress in Scientific culture 1 (2), 1-13, (1976)

[34] П.А.М. Дирак, “Теория позитрона”, Атомное ядро Сборник докладов 1 всесоюзной ядерной конференции, p 139-144, Государственное технико-теоретическое издательство Ленинград-Москва (1934) 
[35] E. Stueckelberg, "La signification du temps propre en mécanique ondulatoire.", Helv. Phys. Acta 14, pp. 322-323. (1941),

[36] R. Feynman, "The Theory of Positrons", Phys. Rev. 76 (76): 749. (1949).

[37] L. Landau, E. Lifshic, “The Classical Theory of Fields”, Fourth Edition: Volume 2 (Course of Theoretical Physics Series), Butterworth-Heinemann; 4 edition (1980)

[38] P. A. M. Dirac, "Quantised singularities in the electromagnetic field", Proc. Roy. Soc. Lond., A133:60-72, (1931).

[39] K.A. Milton, “Theoretical and experimental status of magnetic Monopoles", Review article, e-print arXiv (2006)

[40] R. N. van de Vis, J, E. I. Sfakianakis, et, al. "Nonlinear Dynamics of Preheating after Multifield Inflation with Nonminimal Couplings”, Phys. Rev. Lett. 123, 171301 (2019)

[41] A. H. Guth, "The Inflationary Universe: A Possible Solution To The Horizon And Flatness Problems," Phys. Rev. D 23, 347 (1981).

[42] A. Linde, "Inflationary Cosmology after Planck 2013", e-print https://arxiv.org/abs/1402.0526 (2014)

[43] H. Seeliger, Ueber das Newton'sche Gravitationsgesetz. Astronomische, Nachrichten, 1895 , N. 3273 v. 137 (9), 129-136.

[44] L. Boyle, K. Finn and N. Turok, “CPT-Symmetric Universe”, Phys. Rev. Lett. 121, 251301 (2018).

[45] G.E. Volovik, "Comment to the CPT-symmetric Universe: Two possible extensions", eprint https://arxiv.org/abs/1902.0758419

[46] A.K. Singal, "Solar system peculiar motion from the Hubble diagram of quasars and testing the Cosmological Principle arXiv:2107.0939 [astro-ph.CO]

\title{
Appendix A
}

\author{
Roland E. Allen and Suzy Lidström \\ "Life, the universe, and everything - 42 fundamental Questions" [17]
}

\section{Gravitational and cosmological mysteries}

2.1. The cosmological constant problem

- This problem is outside physics, more see in the main text.

2.2. The dark energy problem

- this problem is outside physics, more see in the main text.

2.3. Regularization of quantum gravity 
- this problem is essentially clarified, and corresponding experiments are suggested, see the main text.

2.4. Black hole entropy and thermodynamics

- no comments.

2.5. Black hole information processing

- no comments.

2.6. Cosmic inflation (or an inflation-like scenario)

- this problem is essentially clarified, see the main text.

2.7. Cosmological survival of matter (and not antimatter)

- this problem is possibly principally solved, see the main text.

2.8. Composition of dark matter

- this problem is possibly rationally elaborated, see the main text.

\section{Understanding and going beyond the Standard Model of particle physics}

3.1. Origin of family replication

- this problem is outside physics, more see in the main text.

3.2. Origin of particle masses

- this problem is outside physics, more see in the main text.

3.3. Supersymmetry and the hierarchy problems

- this problem rather probably really does not exist in physics.

3.4. Explanation of the fundamental grand unified gauge group

- with a rather large probability the "grand unification" problem does not exist as a physical problem, and the fundamental Nature forces are practically independent of each other. Though, at high energies the running of particles' algorithms are essentially deformed, so in such cases an interference of the forces is possible. More see in the main text.

\subsection{Potential violation of Lorentz or CPT invariance}

- the Lorentz transformations are completely valid on macro scale, where the transformations link macro objects "inertial reference frames" and the Voigt-Lorentz decrement can be formed, and they are completely valid only if the macro system of frame instruments and studied bodies is rigid. If that is not so, application of the transformations is limited, more see in the main text.

3.6. Apparent marginality of the Higgs self-coupling, and stability of our universe

- no comment

3.7. Quark confinement and related issues

- no comments

3.8. Phases of quantum chromodynamics and general systems with nonabelian gauge 
interactions

- no comments

\subsection{Additional undiscovered particles}

- no comments

3.10. The unlimited future of astrophysics

- see the main text.

\section{The exotic behavior of condensed matter and quantum systems}

4.1- 4.6 - no comments

\section{Deep issues}

5.1. Higher dimensions, with geometry and topology of an internal space

Matter's utmost fundamental and universal spacetime is the absolute, fundamentally "flat", [5]4D Euclidian spacetime with the utmost fundamental and universal metrics $(c \tau, X, Y, Z, c t)$, where the dimensions relate to corresponding degrees of freedom at FLE states changes. Other "topologies" with a large probability would be purely artificial theoretical constructions. More see in the main text.

\subsection{Validity of the multiverse idea and the anthropic principle}

- "Multiverse", as it was firstly introduced in physics as a version of quantum mechanics interpretation, seem a rather unphysical phenomenon, at least by the energy reason. Even to create the observed one Matter it was necessary to spend a practically unbelievable portion of energy, to create infinite "number" of Matters would need spending an infinitely unbelievable portion. "Anthropic principle" hence has no physical applications, even that would be a rational principle outside physics.

\subsection{Geometry and topology of external spacetime}

- Matter, and the Matter's spacetime, indeed exist as a part of the spacetime of the absolutely fundamental and absolutely infinite "Information" Set, which - the Set's spacetime - has at least infinite "number" of space dimensions and at least one "true time" dimension. However now humans know almost nothing about the Set's content and corresponding "external spacetime" besides that it is composed in accordance with the common definitions of the "Logos" elements "Space" and "Time", more see in the main text.

5.4. Origin and fate of the universe.

5.5. What is the origin of spacetime, why is spacetime four-dimensional, and why is time different from space?

- see the main text.

5.6. Origin of Lorentz invariance and Einstein gravity

- see $[5 a]$.

5.8. Origin and interpretation of quantum mechanics and quantum fields 
- see the main text.

5.9. Mathematical consistency

- Matter is a rather simple informational system based on a simple binary reversive logics and a rather small set of universal fundamental laws/links/constants, and where exchange by information happens as exchange of completely true information. Such system is so can be, and so is, effectively described by mathematics, and mathematics is indeed an extremely effective tool. But hardly more than a tool.

5.10. Connection between the formalism of physics and the reality of human experience

- see the main text.

\section{Potential for breakthroughs in techniques and technology}

6.1. -6.2

- no comments.

\section{Life}

7.1. What is life?

- see the main text.

7.2. How did life on Earth begin - and how did complex life originate?

- see the main text.

7.3. How abundant is life in the universe, and what is the destiny of life?

- to answer the question there is no any reliable information now; and that seems rather possibly isn't too actual.

7.4. How does life solve problems of seemingly impossible complexity?

- life does not solve this problem as a critical fundamental task, more see in the main text..

7.5. Can we understand and cure the diseases that afflict life?

- that is not a fundamentally irresolvable problem in most cases.

7.6. What is consciousness?

- see the main text.

\section{Who will solve the biggest problems?}

- see the main text. 